\title{
Variation and diversity of the breakpoint sequences on 4AL for 4AL/5AL translocation in Triticum
}

\begin{tabular}{|r|l|}
\hline Journal: & Genome \\
\hline Manuscript ID & gen-2018-0060.R1 \\
\hline Manuscript Type: & Article \\
\hline Date Submitted by the Author: & $30-$ May-2018 \\
\hline Complete List of Authors: & $\begin{array}{l}\text { Luo, Wei; Triticeae Research Institute, Sichuan Agricultural University } \\
\text { Qin, Na-Na; Triticeae Research Institute, Sichuan Agricultural University } \\
\text { Mu, Yang; Triticeae Research Institute, Sichuan Agricultural University } \\
\text { Tang, Huaping; Triticeae Research Institute, Sichuan Agricultural University } \\
\text { Deng, Mei; Triticeae Research Institute, Sichuan Agricultural University } \\
\text { Liu, Yaxi; Triticeae Research Institute, Sichuan Agricultural University } \\
\text { Chen, Guangdeng; College of Resources, Sichuan Agricultural University } \\
\text { Jiang, Qian-Tao; Sichuan Agricultural University, Triticeae Research } \\
\text { Institute } \\
\text { Chen, Guo-Yue; Triticeae Research Institute, Sichuan Agricultural } \\
\text { University } \\
\text { Wei, Yuming; Triticeae Research Institute of Sichuan Agricultural } \\
\text { University, } \\
\text { Zheng, Youliang; Sichuan Agricultural University, } \\
\text { Lan, Xiu-Jin; Sichuan Agricultural University, Triticeae Research Institute } \\
\text { Ma, Jian; Triticeae Research Institute, Sichuan Agricultural University }\end{array}$ \\
\hline Keyword: & 4AL/5AL translocation, Triticum, breakpoint, diversity \\
\hline Is the invited manuscript for \\
consideration in a Special \\
Issue? :
\end{tabular} \begin{tabular}{l} 
N/A \\
\hline
\end{tabular}


1 Variation and diversity of the breakpoint sequences on 4AL for

2 4AL/5AL translocation in Triticum

3 Wei Luo ${ }^{1,+}$, Nana Qin $^{1,+}$, Yang $\mathrm{Mu}^{1}$, Huaping Tang ${ }^{1}$, Mei Deng ${ }^{1}$, Yaxi Liu ${ }^{1}$,

4 Guangdeng Chen ${ }^{2}$, Qiantao Jiang ${ }^{1}$, Guoyue Chen ${ }^{1}$, Yuming $\mathrm{Wei}^{1}$, Youliang Zheng ${ }^{1}$,

5 Xiujin $\operatorname{Lan}^{1, *}$, Jian $\mathrm{Ma}^{1, *}$

6

$7{ }^{1}$ Triticeae Research Institute, Sichuan Agricultural University, Chengdu, Sichuan, 8 611130, China

$9{ }^{2}$ Institute of Ecological and Environmental Sciences, Sichuan Agricultural University,

10 Wenjiang, Chengdu, 611130, China

$11+$ Contributed equally to this paper.

$12 *$ Authors for correspondence:

Dr. Jian Ma, E-mail: jianma@ sicau.edu.cn

Dr. Xiujin Lan, E-mail: lanxiujin@163.com

Tel: +86 28 86293115; Fax: +86 2882650350

The e-mail addresses of the other co-authors: thisisl.w@foxmail.com (Wei Luo), 2249183768@qq.com (Nana Qin), Julinka0024@163.com (Yang $\mathrm{Mu})$, 707952940@qq.com (Huaping Tang), dengmei105@163.com (Mei Deng), yaxi.liu@outlook.com (Yaxi Liu), gdchen@sicau.edu.cn (Guangdeng Chen), qiantaojiang@sicau.edu.cn (Qiantao Jiang), guoyuech74@hotmail.com (Guoyue 
25 Abstract: The translocation of $4 \mathrm{AL} / 5 \mathrm{AL}$ in Triticum, occurred before the 26 differentiation of T. urartu and einkorn, is an important chromosomal rearrangement 27 incident. Recently the first identification of breakpoint sequence on 4AL for this translocation provides the chance to analyze the variation and diversity of this breakpoint in Triticum. In this study, the breakpoint regions of 52 accessions from 21 species were isolated and further characterized. The sequences were divided into 12 types based on length differences ranging from 2009 to $2552 \mathrm{bp}$. Cluster analysis showed that they were further divided into three groups. Interesting evolutionary relationships among a few of the species were observed and discussed. Multiple sequence alignment of the 52 sequences detected 13 insertion and deletion length polymorphism (InDel) and 101 single nucleotide polymorphism (SNP). Besides, several species or accession specific SNP or InDel were also identified. Based on BLAST analysis of the conserved sequences, the breakpoint was narrowed down to a $125 \mathrm{bp}$ fragment. Taken together, the results obtained in this study enrich our understanding of the breakpoints and will be helpful for identification of other breakpoints in wheat.

Key words: 4AL/5AL translocation, Triticum, breakpoint, diversity 


\section{Introduction}

Chromosomal rearrangements play an important role in the evolution of various species (Morrow and Cooper 2012; Sankoff and Nadeau 2003). Previous studies have identified quite a few of chromosomal rearrangements in wheat (Berkman et al. 2012; Devos et al. 1995; Ma et al. 2015a; Ma et al. 2013; Ma et al. 2014; Ma et al. 2015b; Nelson et al. 1995). The most well-known of such rearrangements are the non-homoeologous translocations (4A-5A-7B) occurred between chromosomes 4A, $5 \mathrm{~A}$ and $7 \mathrm{~B}$ in the hexaploid wheat (Triticum aestivum L., $2 \mathrm{n}=6 \mathrm{x}=42$, genomes $\mathrm{AABBDD})$. It is widely believed that the $4 \mathrm{AL} / 5 \mathrm{AL}$ translocation occurred at the diploid level and the 4A/7B translocation must have occurred at the tetraploid level as it is also present in $T$. durum $(2 \mathrm{n}=4 \mathrm{x}=28$, genomes AABB) (Devos et al. 1995). Most recently, the breakpoints of the $4 \mathrm{AL} / 5 \mathrm{AL}$ translocation were identified and the $4 \mathrm{AL}$ junction was narrowed down to $\sim 1000$ bp in $T$. monococcum, T. urartu and $T$. aestivum (Li et al. 2016).

It was documented that more than ten species or subspecies were classified into Triticum (Briggle and Reitz 1963; Dong 1979). Scientists have long been focused on studying the origin of the species. To date, geneticists still hold different opinions on the origins of some species. For example, Yang et al. (1992b) thought that Tibetan semi-wild wheat (T. aestivum ssp. tibetanum) is the progeny of tetraploid wheat and Chinese Aegilops tauschii Cosson, while Tsunewaki et al. (1990) suggested it is the offspring of Tibetan common wheat. Chen et al. (1985) thought Xinjiang rice wheat ( $T$. petropavlovskyi) was evolved from natural hybridization of $T$. polonicum with common wheat and then derived from the backcross with common wheat, yet Yang et al. (1992a) and Chen (1999) inferred it came from the distant hybridization descendant of $T$. polonicum and A. tauschii. In sunflower, chromosomal rearrangements play a substantial role in adaptive divergences, species integrity and genetic identity in extensive hybridization and gene flow (Strasburg et al. 2009). Thus, analysis of the breakpoints in Triticum could benefit our understanding of the origin 
72

and evolution of some species.

Genetic variation is directly involved into the environmental adaptability of plant (Nevo et al. 1982; Prentis et al. 2008). In Triticum species, the evolutionary processes might cause genetic variations of a given gene or a sequence (Gautier et al. 2000; Huang et al. 2002b; Ma et al. 2017). Therefore, analysis of different genes could help to understand the origin and evolution of the Triticum species. For example, Xu et al. (2009) detected 37 high molecular weight glutenin subunits alleles in diploid, tetraploid and hexaploid wheat, and they presumed that $T$. carthlicum might experience a different evolutional way with other tetraploid wheat given their owned special $1 A y$ alleles. Huang et al. (2002a) analyzed the divergence and evolution of wheat and other grasses based on the genes encoding acetyl-CoA carboxylase and plastid 3-phosphoglycerate kinase.

In the present study, the breakpoint regions of $4 \mathrm{AL} / 5 \mathrm{AL}$ chromosomal translocation on 4AL were sequenced and analyzed in diploid, tetraploid and hexaploid species of Triticum. Quite a few of insertion and deletion length polymorphism (InDel) and single nucleotide polymorphism (SNP) were detected among species. The relationships among different species were also analyzed based on the breakpoint sequences. The breakpoint interval was narrowed to $125 \mathrm{bp}$ based on the chromosome arm location pattern of the flanking sequences.

\section{Materials and Methods}

\section{Plant materials and DNA isolation}

A total of 52 Triticum accessions from 21 species were used in this study (Table 1). All of the accessions were planted at the experimental farm of the Triticeae Research Institute, Sichuan Agricultural University, China in 2015-2016. The fresh leaves of the plants at seedling stage were harvested and ground into powder with liquid nitrogen, and then the genomic DNA was extracted from it by using the cetyltrimethylammonium bromide method (Li et al. 2012) and stored at $-20{ }^{\circ} \mathrm{C}$ for the 
99

100

101

102

103

104

105

106

107

108

109

110

111

112

113

114

115

116

117

118

119

120

121

122

123

following procedure.

\section{Sequencing of the breakpoint}

Two pairs of universal primers (P1 and P2) were designed to amplify the breakpoint region of 4AL based on the sequences of T. urartu, T. monococcum and T. aestivum (Li et al. 2016), and the reference sequences were shown in Table S1. The forward primer F1 of primer P1, 5'- CAGAGCATTTGAAATATCA-3', was closed to the 3' part of $W D 3 L$, and the reverse primer R1, 5'-GCACCATTTCATTCTCCATTGC-3', was connected with 3' part of PMEIL (Fig. 1A). The forward primer F2 of primer P2, 5'-CATGGACGGCATACACAGAG-3', and the reverse primer R2, 5'-GCTGGTGGTTGATGTGATTG-3', were connected with 3' part of WD3L and PMEIL (Fig. 1A), respectively. The primers were synthesized by Tsingke Biological Technology (Chengdu, China; http://www.tsingke.net/).

The PCR was carried out in a $50 \mu \mathrm{l}$ mixture containing $25 \mu \mathrm{l} 2 \times$ TSINGKE Master Mix (blue) (Tsingke Biological Technology), $5 \mu$ DNA template $(\sim 200 \mathrm{ng} / \mu \mathrm{l}), 2.5 \mu \mathrm{l}$ of each primer $(10 \mu \mathrm{M})$ and $15 \mu \mathrm{ddH} 2 \mathrm{O}$. At least five independent PCR mixtures for each accession were collected and sequenced in both directions by Tsingke Biological Technology (Chengdu, China). The sequences amplified by P1 and P2 from a given accession were assembled by DNAMAN 6.0 (Lynnon Biosoft, USA).

\section{Sequence analysis}

The analysis of breakpoint sequence and multiple sequence alignment were carried out by DNAMAN 6.0. The construction of bootstrap consensus tree and analysis of nucleotide polymorphism (including InDel and SNP) were performed by MEGA 6.0 (Tamura et al. 2013).

\section{Results}

Isolation of breakpoint sequences 
The expected fragments of breakpoint sequences for all the accessions in the present study were successfully isolated using two sets of primers (Fig. 1B and 1C). Multiple sequence alignments showed that all the obtained sequences belong to the breakpoint on $4 \mathrm{AL}$ for $4 \mathrm{AL} / 5 \mathrm{AL}$ translocation based on the result of Li et al. (2016). Sequencing and assembly identified 12 types of sequences with different length termed from Type I to Type XII (Table 2). The sequence length of these groups ranged from $2009 \mathrm{bp}$ (XII) to 2552 bp (I) (Table 2). The obtained breakpoint sequences of seven species belong to Type II, while only one species belong to each of Type I, V, VII, IX, X and XII (Table 2).

The sequence similarity was $10.32 \%$ for all of the 52 accessions, and that for diploid, tetraploid and hexaploid wheat were $71.66,84.02$ and $94.58 \%$, respectively. In addition, we found that Type VII, VIII and IX were constituted by diploid wheat only; tetraploid wheat was classified to Type II (only $T$. persicum), III (such as $T$. dicoccoides and T. persicum), IV (T. dicoccoides and T. dicoccum), VI (including $T$. durum and T. araraticum), XI (T. polonicum and T. paleo-colchicum) and XII (only T. timopheevii); and hexaploid wheat were categorized to Type I (only $T$. petropavlovishyi), II (i.e. T. zhukovskyi, T. compactum, T. macha, T. sphaerococum and T. vavilovii), III (including T. compactum, T. yunnanense and T. tibetanum) and and X (only T. zhukovskyi) (Table 2). Furthermore, both Type II and III were consisted by tetraploid and hexaploid wheat. These results likely suggested that several types of breakpoint sequences were present in Triticum.

\section{SNPs and InDels in breakpoint region}

One hundred and one single nucleotide substitutions and 13 InDels ranging from 1 to 275 bp were detected among all the sequences (Fig. S1). In diploid wheat, 47 single nucleotide substitutions and 8 InDels were detected. In tetraploid wheat, there were 29 single nucleotide substitutions and 6 InDel regions with length of 1 (two loci), 2, 6, 60 and $105 \mathrm{bp}$. Furthermore, a total of 62 single nucleotide substitutions and $10 \mathrm{InDel}$ regions ranging from 1 (6 loci), 6, 101, 105 and $275 \mathrm{bp}$ in length were obtained in 
hexaploid wheat (Fig. S1).

153

154

155

156

157

158

159

160

161

162

163

164

165

166

167

168

169

170

171

172

173

174

175

176

177

178

Interestingly several significant diversities were observed: (1) some InDels were detected in several species only, for example, four inserted and one deleted sequences (InDel-A, B, D, F and K) were detected in T. beoeticum and T. monococcum only; (2) several InDels could be observed in a single species, e.g., there were two InDels in the amplified sequences of 4 accessions of T. polonicum; (3) genotype specificity InDel was also detected, for instance, a unique " $G$ " insertion was present in the $495^{\text {th }}$ bp of accession PI393496 for T. monococcum) (Fig. S1).

\section{Cluster analysis of breakpoint sequences from diploid, tetraploid and hexaploid wheat}

To better understand the relationships of the breakpoint sequences for the accessions, a bootstrap consensus tree was generated (Fig. 2). As shown, all the sequences could be divided into three main groups mainly based on the ploidy of the materials. Group I was consisted of einkorn, including T. boeoticum and T. monococcum. Group II was most complicated and it was mainly composed by tetraploid species. Interestingly, $T$. urartu and T. zhukovskyi PI355706 belong to Group II. Most of the hexaploid wheat (except T. zhukovskyi PI355706), all the T. persicum and T. dicoccoides AS838 were classified as Group III. It was found that some accessions from the same species or sibling species were categorized together meaning that these accessions may be closely related. For example, T. boeoticum and T. monococcum were classified as one group with 100 of bootstrap value. However, some accessions from a single species, were fallen into different clades. For example, the three accessions of $T$. vavilovii were in three different clades. In addition, the bootstrap values for many clades were less than 50 reducing the confidence of evolutionary relationships for the species.

\section{Narrowing down the breakpoint interval}

In order to identify the accurate breakpoint on $4 \mathrm{AL}$ for the $4 \mathrm{AL} / 5 \mathrm{AL}$ translocation in wheat, the conserved fragments in the multiple sequence alignment of the Triticum 
were used for assigning the International Wheat Genome Sequencing Consortium $\begin{array}{lllll}\text { Reference } & \text { Sequence } & \text { v1.0 } & \text { (IWGSC } & \text { RefSeq }\end{array}$ (https://wheat-urgi.versailles.inra.fr/Seq-Repository/Assemblies). We found that fragment A was similar to sequences on $4 \mathrm{AL}, 4 \mathrm{BL}$ and $4 \mathrm{DL}$, while fragments $\mathrm{G}$ had similarity to sequences on 4AL, 5BL and 5DL (Fig. 3). No hit was identified for fragment $\mathrm{C}, \mathrm{E}$ and $\mathrm{F}$, and fragment $\mathrm{B}$ and $\mathrm{D}$ were similar to sequences on $4 \mathrm{AL}$. There is a significant difference for the sequence relationship between fragments $A$ and $G$, we thus speculated that the breakpoint is likely located in the $125 \mathrm{bp}$ between fragment $\mathrm{A}$ and $\mathrm{G}$ (Fig. 3).

\section{Discussion}

\section{Breakpoint sequences based cluster analysis of the origin and evolution of} Triticum

Cluster analysis indicated that $T$. urartu and the species with G genome including $T$. timopheevii (AAGG), T. araraticum (AAGG) and T. zhukovskyi PI355706 $\left(\mathrm{AAA}^{\mathrm{m}} \mathrm{A}^{\mathrm{m}} \mathrm{GG}\right)$ were clustered together with a high bootstrap value (77), which means that $T$. urartu and T. timopheevii or T. araraticum might be involved in the origin of $T$. zhukovskyi. This was consistent with previous studies pointing that T. timopheevii was the ancestor of T. zhukovskyi (Talbert et al. 1991) and the ancestor of T. urartu was the donor of A genome of tetraploid and hexaploid wheat (Allaby et al. 1999).

As T. zhukovskyi was suggested to have arisen via hybridization of T. timopheevii originated from crossing of Ae. speltoides with T. urartu (Allaby and Brown 2000; Dvorák et al. 1993; Kilian et al. 2007) and T. monococcum with subsequent chromosome doubling (Bowden 1959; Dvorák et al. 1993), two different amplified sequences orthologous to those of T. urartu (A) and T. monococcum $\left(\mathrm{A}^{\mathrm{m}}\right)$, respectively, could be obtained. Using a pair of primers spanning an $\sim 2.4 \mathrm{~kb}$ fragment, Li et al. (2016) amplified two weak bands in T. zhukovskyi corresponding to the A and $\mathrm{A}^{\mathrm{m}}$ genome. In this study, a single band similar to that from T. urartu was obtained 
only in T. zhukovskyi by the designed primers. We thus speculate that the sequence on $\mathrm{A}^{\mathrm{m}}$ genomes of $T$. zhukovskyi may be obtained by newly designed primers.

T. petropavlovishyi, T. tibetanum and T. yunnanense are landraces found only in China. They showed similar original characters including brittle rachis and hard-threshability. It was suggested they should have similar evolutionary process (Jiang et al. 2014; Wang et al. 2005; Yang 1992). Multiple sequences alignments in this study showed that a 60 bp fragment (InDel-J) was missing from T. petropavlovishyi (Fig. S1). Only T. tibetanum and T. yunnanense were clustered in one clade (Fig. 2) showing a farther genetic relationship with $T$. petropavlovishyi. This was consistent with the results of taxonomic classifications (Chen et al. 2016; Ward et al. 1998; Yang et al. 1993; Yang et al. 1992b) based on morphological traits, cytogenetics, ecogeographical origins and restriction fragment length polymorphism (RFLP) analysis indicating that $T$. tibetanum and T. yunnanense might experience a similar origin and (or) evolution compared to T. petropavlovishyi.

It was widely believed that $T$. dicoccum was the A and B donors of $T$. spelta (Peng et al. 2011). Here we found that T. tibetanum and T. yunnanense were clustered with $T$. dicoccoides (Fig. 2), likely suggesting that T. tibetanum and T. yunnanense may be derived from $T$. dicoccoides. This possible evolutionary difference between $T$. spelta and T. tibetanum and T. yunnanense may contrite to their different brittle rachis characters (Wang and Zheng 2004).

Different from previous evolutionary analyses based on phenotype characteristics (Peng et al. 2011), chromosome paring (El Baidouri et al. 2017; Feldman and Levy 2005; Peng et al. 2011), and gene similarity (Novoselskaya-Dragovich et al. 2018; Peng et al. 2011; Yan et al. 2017), we here conducted cluster analysis of the main species in Triticum by employment of the breakpoint sequences on $4 \mathrm{AL}$ for $4 \mathrm{AL} / 5 \mathrm{AL}$ translocation. We indeed observed a few of evolutionary relationships among some species being consistent with those detected in previous studies. For example, in addition to those discussed above, we also found that both einkorn and emmer wheat 
were expectedly clustered together closely (Fig. 2). However, quite a few of the detected relationships among some species were different from those previously observed by traditional classification. For example, T. persicum, T. spelta and $T$. vavilovii were categorized into more than one clades being different from traditional classification (Fig. 2). The reason is likely that the breakpoint sequences suffered from extensive, intense and unidirectional variations as the environment changed after divergence from the ancestor. These sequence polymorphisms masked the real evolutionary picture of different species in Triticum. We thus suggested that the ability of breakpoints based analysis of evolution could be limited. The identification of other breakpoints for $4 \mathrm{~A}-5 \mathrm{~A}-7 \mathrm{~B}$ translocation is yet to be further employed in evolutionary analysis.

\section{Utilization potential of the detected SNPs and InDels}

In the genetic research of wheat, one of the most important jobs is to map genes or major quantitative trait loci (QTL). The development of molecular markers based on SNPs or InDels plays vital function in construction of genetic maps, especially fine mapping of genes or QTL. For example, Lu et al. (2016) developed high-resolution melting (HRM) markers to map powdery mildew resistance gene PmPB74; Qureshi et al. (2017) designed kompetitive allele specific PCR (KASP) marker to link rust resistance genes $\operatorname{Yr} 47$ and $\operatorname{Lr} 52$; The InDel and cleaved amplified polymorphic sequence (CAPS) markers were developed to study the grain weight gene $T G W-7 A$ in wheat (Hu et al. 2016). Abundant of SNPs and InDels were detected in this study, and some of them were specific to a given genotype or species (Fig. S1). For example, InDel-A and InDel-K were inserted and deleted in both $T$. beoeticum and $T$. monococcum, respectively; and a $60 \mathrm{bp}$ fragment, i.e. InDel-J, was only deleted from T. petropavlovishyi. Thus, the SNPs and InDels identified here may be useful for developing molecular markers to map genes or QTLs flanking the breakpoint on 4A of wheat. 
262

263

264

265

266

267

268

269

270

271

272

273

274

275

276

277

278

279

280

281

282

283

284

285

286

287

288

289

290

291

292

293

identification of breakpoints

By using Aegilops tauschii, CS chromosome survey sequences, T. monococcum and T. urartu genomic sequences deposited in public database, the 4AL junction of the breakpoint for the $4 \mathrm{AL} / 5 \mathrm{AL}$ translocation was recently identified, and the breakpoint was located in an $\sim 1000$ bp of interval ( $\mathrm{Li}$ et al. 2016). Here, the isolated conserved sequences from 21 species of Triticum flanking the 4AL breakpoint were used as queries for searching the recent assemblies of IWGSC RefSeq v1.0 and the breakpoint interval was narrowed down to a $125 \mathrm{bp}$ fragment on 4AL. It is believed that other breakpoints for 4A-5A-7B translocations could be more easily identified with the publication of updated CS chromosome assembly sequences.

\section{Acknowledgements}

This publication is based upon work supported by the projects from the National Key Research and Development Program of China (2017YFD0100900), the Applied Basic Research (2016JY0010), and International Science and Technology Cooperation and Exchanges (2017HH0076) Programs of Science and Technology Department of Sichuan Province. We thanks Dr. Bihua Wu, Dr. Zehong Yan, and Dr. Lianquan Zhang, from Triticeae Research Institute, Sichuan Agricultural University, China for providing the materials used in this study. We appreciate the anonymous referees for critical reading of the manuscript.

Conflict of interest All authors read and agreed with the final manuscript and have no conflicts of interest.

\section{Reference}

Allaby, R.G., Banerjee, M., and Brown, T.A. 1999. Evolution of the high molecular weight glutenin loci of the A, B, D, and G genomes of wheat. Genome, 42(2): 296-307. doi: 10.1139/g98-114.

Allaby, R.G., and Brown, T.A. 2000. Identification of a 5S rDNA spacer type specific Triticum urartu and wheats containing the T. urartu genome. Genome, 43(2): 250-254. doi: $10.1139 / \mathrm{g} 99-122$.

Berkman, P.J., Skarshewski, A., Manoli, S., Lorenc, M.T., Stiller, J., Smits, L. et al. 2012. Sequencing wheat chromosome arm 7BS delimits the 7BS/4AL translocation and reveals homoeologous gene conservation. Theor. Appl. Genet. 124(3): 423-432. 
doi: 10.1007/s00122-011-1717-2.

Bowden, W.M. 1959. The taxonomy and nomenclature of the wheats, barleys, and ryes and their wild relatives. Can. J. Bot. 37(4): 657-684. https://doi.org/10.1139/b59-053

Briggle, L.W., and Reitz, L.P. 1963. Classification of Triticum species and of wheat varieties grown in the United States. U.S. Dept. of Agriculture, Washington.

Chen, Q.-F. 1999. Discussion on origin of Chinese endemic wheat. Guizhou Agric. Sci. 27(1): 20-25.

Chen, Q., Song, J., Du, W.-P., Xu, L.-Y., and Yu, G.-R. 2016. Possible origin of Triticum petropavlovskyi based on cytological analyses of crosses between $T$. petropavlovskyi and tetraploid, hexaploid, and synthetic hexaploid (SHW-DPW) wheat accessions. Span. J. Agric. Res. 14(4): e0713. doi: 10.5424/sjar/2016144-8476. Chen, Q., Sun, Y.-Z., and Dong, Y.-S. 1985. Cytogenetical studies on interspecific hybrids of Xinjiang Wheat. Acta Agron. Sinica. 11(1): 23-29.

Devos, K.M., Dubcovsky, J., Dvořák, J., Chinoy, C.N., and Gale, M.D. 1995. Structural evolution of wheat chromosomes $4 \mathrm{~A}, 5 \mathrm{~A}$, and $7 \mathrm{~B}$ and its impact on recombination. Theor. Appl. Genet. 91(2): 282-288. doi: 10.1007/BF00220890.

Dong, Y.S. 1979. Race species in Triticum and their utilization in breeding. Sci. Agr. Sinica 12(3): 1-7.

Dvorák, J., Terlizzi, P., Zhang, H.B., and Resta, P. 1993. The evolution of polyploid wheats: identification of the A genome donor species. Genome, 36(1): 21-31. doi: 10.1139/g93-004.

El Baidouri, M., Murat, F., Veyssiere, M., Molinier, M., Flores, R., Burlot, L. et al. 2017. Reconciling the evolutionary origin of bread wheat (Triticum aestivum). New Phytol. 213(3): 1477-1486. doi: 10.1111/nph.14113.

Feldman, M., and Levy, A.A. 2005. Allopolyploidy - a shaping force in the evolution of wheat genomes. Cytogenet. Genome Res. 109(1-3): 250-258. https://doi.org/10.1159/000082407

Gautier, M.-F., Cosson, P., Guirao, A., Alary, R., and Joudrier, P. 2000. Puroindoline genes are highly conserved in diploid ancestor wheats and related species but absent in tetraploid Triticum species. Plant Sci. 153(1): 81-91. doi: http://dx.doi.org/10.1016/S0168-9452(99)00258-7.

Hu, M.J., Zhang, H.P., Liu, K., Cao, J.J., Wang, S.X., Jiang, H. et al. 2016. Cloning and Characterization of TaTGW-7A Gene Associated with Grain Weight in Wheat via SLAF-seq-BSA. Front. Plant Sci. 7: 1902. doi: 10.3389/fpls.2016.01902.

Huang, S., Sirikhachornkit, A., Faris, J.D., Su, X., Gill, B.S., Haselkorn, R. et al. 2002a. Phylogenetic analysis of the acetyl-CoA carboxylase and 3-phosphoglycerate kinase loci in wheat and other grasses. Plant Mol. Biol. 48(5): 805-820. doi: 10.1023/a:1014868320552.

Huang, S., Sirikhachornkit, A., Su, X., Faris, J., Gill, B., Haselkorn, R. et al. 2002b. Genes encoding plastid acetyl-CoA carboxylase and 3-phosphoglycerate kinase of the Triticum/Aegilops complex and the evolutionary history of polyploid wheat. Proc. Natl. Acad. Sci. U. S. A. 99(12): 8133-8138. doi: 10.1073/pnas.072223799.

Jiang, Y.-F., Lan, X.-J., Luo, W., Kong, X.-C., Qi, P.-F., Wang, J.-R. et al. 2014. 
Genome-wide quantitative trait locus mapping identifies multiple major loci for brittle rachis and threshability in Tibetan semi-wild wheat (Triticum aestivum ssp. tibetanum Shao). PLoS ONE, 9(12): e114066. https://doi.org/10.1371/journal.pone.0114066 Kilian, B., Ozkan, H., Deusch, O., Effgen, S., Brandolini, A., Kohl, J. et al. 2007. Independent wheat $\mathrm{B}$ and $\mathrm{G}$ genome origins in outcrossing Aegilops progenitor haplotypes. Mol. Biol. Evol. 24(1): 217-227. doi: 10.1093/molbev/msl151.

Li, W., Challa, G.S., Zhu, H., and Wei, W. 2016. Recurrence of chromosome rearrangements and reuse of DNA breakpoints in the evolution of the Triticeae genomes. G3-Genes Genom. Genet. 6(12): 3837-3847. https://doi.org/10.1534/g3.116.035089

Li, W., Gao, Z., Xiao, W., Wei, Y.-M., Liu, Y.-X., Chen, G.-Y. et al. 2012. Molecular diversity of restriction enzyme sites, Indels and upstream open reading frames (uORFs) of 5' untransalted regions (UTRs) of Waxy genes in Triticum L. and Aegilops L. species. Genet. Resour. Crop Ev. 59(8): 1625-1647. https://doi.org/10.1007/s10722-011-9787-1

Lu, Y., Yao, M., Zhang, J., Song, L., Liu, W., Yang, X. et al. 2016. Genetic analysis of a novel broad-spectrum powdery mildew resistance gene from the wheat-Agropyron cristatum introgression line Pubing 74. Planta, 244(3): 713-723. doi: 10.1007/s00425-016-2538-y.

Ma, J., Gao, S., Stiller, J., Jiang, Q.T., Lan, X.J., Liu, Y.X. et al. 2015a. Identification of genes bordering breakpoints of the pericentric inversions on $2 \mathrm{~B}, 4 \mathrm{~B}$, and $5 \mathrm{~A}$ in bread wheat (Triticum aestivum L.). Genome, 58(8): 385-390. doi: 10.1139/gen-2015-0060.

Ma, J., Stiller, J., Berkman, P.J., Wei, Y., Rogers, J., Feuillet, C. et al. 2013. Sequence-based analysis of translocations and inversions in bread wheat (Triticum aestivum L.). PLoS ONE, 8(11): e79329. doi: 10.1371/journal.pone.0079329.

Ma, J., Stiller, J., Wei, Y., Zheng, Y.L., Devos, K.M., Dolezel, J. et al. 2014. Extensive pericentric rearrangements in the bread wheat (Triticum aestivum L.) genotype "Chinese Spring" revealed from chromosome shotgun sequence data. Genome Biol. Evol. 6(11): 3039-3048. doi: 10.1093/gbe/evu237.

Ma, J., Stiller, J., Zheng, Z., Wei, Y., Zheng, Y.L., Yan, G. et al. 2015b. Putative interchromosomal rearrangements in the hexaploid wheat (Triticum aestivum L.) genotype 'Chinese Spring' revealed by gene locations on homoeologous chromosomes. BMC Evol. Biol. 15: 37. doi: 10.1186/s12862-015-0313-5.

Ma, J., Yang, Y., Luo, W., Yang, C., Ding, P., Liu, Y. et al. 2017. Genome-wide identification and analysis of the MADS-box gene family in bread wheat (Triticum aestivum L.). PLoS ONE, 12(7): e0181443. doi: 10.1371/journal.pone.0181443.

Morrow, J.D., and Cooper, V.S. 2012. Evolutionary effects of translocations in Bacterial genomes. Genome Biol. Evol. 4(12): 1256-1262. doi: 10.1093/gbe/evs099. Nelson, J.C., Sorrells, M.E., Van Deynze, A.E., Lu, Y.H., Atkinson, M., Bernard, M. et al. 1995. Molecular mapping of wheat: major genes and rearrangements in homoeologous groups 4, 5, and 7. Genetics, 141(2): 721.

Nevo, E., Golenberg, E., Beiles, A., Brown, A., and Zohary, D. 1982. Genetic diversity and environmental associations of wild wheat, Triticum dicoccoides, in 
Israel. Theor. Appl. Genet. 62(3): 241-254. https://doi.org/10.1007/BF00276247

Novoselskaya-Dragovich, A.Y., Fisenko, A.V., Konovalov, F.A., Mitrofanova, O.P., Shishkina, A.A., and Kudryavtsev, A.M. 2018. Analysis of genetic diversity and evolutionary relationships among hexaploid wheats Triticum L. using LTR-retrotransposon-based molecular markers. Genet. Resour. Crop Ev. 65(1): 187-198. doi: 10.1007/s10722-017-0520-6.

Peng, J.H., Sun, D., and Nevo, E. 2011. Domestication evolution, genetics and genomics in wheat. Mol. Breed. 28(3): 281-301. doi: 10.1007/s11032-011-9608-4.

Prentis, P.J., Wilson, J.R.U., Dormontt, E.E., Richardson, D.M., and Lowe, A.J. 2008. Adaptive evolution in invasive species. Trends Plant Sci. 13(6): 288-294. doi: http://dx.doi.org/10.1016/j.tplants.2008.03.004.

Qureshi, N., Bariana, H., Forrest, K., Hayden, M., Keller, B., Wicker, T. et al. 2017. Fine mapping of the chromosome 5B region carrying closely linked rust resistance genes $Y r 47$ and Lr52 in wheat. Theor. Appl. Genet. 130(3): 495-504. doi: 10.1007/s00122-016-2829-5.

Sankoff, D., and Nadeau, J.H. 2003. Chromosome rearrangements in evolution: From gene order to genome sequence and back. Proc. Natl. Acad. Sci. U. S. A. 100(20): 11188-11189. doi: 10.1073/pnas.2035002100.

Strasburg, J.L., Scotti-Saintagne, C., Scotti, I., Lai, Z., and Rieseberg, L.H. 2009. Genomic patterns of adaptive divergence between chromosomally differentiated sunflower species. Mol. Biol. Evol. 26(6): 1341-1355. doi: 10.1093/molbev/msp043.

Talbert, L.E., Magyar, G.M., Lavin, M., Blake, T.K., and Moylan, S.L. 1991. Molecular evidence for the origin of the S-derived genomes of polyploid Triticum species. Am. J. Bot. 78(3): 340-349. doi: 10.2307/2444956

Tamura, K., Stecher, G., Peterson, D., Filipski, A., and Kumar, S. 2013. MEGA6: molecular evolutionary genetics analysis version 6.0. Mol. Biol. Evol. 30(12): 2725-2729. doi: 10.1093/molbev/mst197.

Tsunewaki, K., Yamada, S., and Mori, N. 1990. Genetical studies on a Tibetan semi-wild wheat, Triticum aestivum ssp. tibetanum. Jpn. J. Genet. 65(5): 353-365. doi: 10.1266/jjg.65.353.

Wang, H., Wang, X.e., Chen, P., and liu, D. 2005. Analysis of gliadin allele composition at Gli-1 and Gli-2 loci and genetic diversity in Yunnan, Tibetan and Xinjiang wheats. J. Triticeae Crops. 25(6): 34-39.

Wang, Z.Q., and Zheng, Y.L. 2004. Advances on the study of Tibetanum Semi-wild Wheat. J. Triticeae Crops. 24(4): 133-135.

Ward, R.W., Yang, Z.L., Kim, H.S., and Yen, C. 1998. Comparative analyses of RFLP diversity in landraces of Triticum aestivum and collections of T. tauschii from China and Southwest Asia. Theor. Appl. Genet. 96(2): 312-318. doi: $10.1007 / \mathrm{s} 001220050742$.

Xu, L.-L., Li, W., Wei, Y.-M., and Zheng, Y.-L. 2009. Genetic diversity of HMW glutenin subunits in diploid, tetraploid and hexaploid Triticum species. Genet. Resour. Crop Ev. 56(3): 377-391. doi: 10.1007/s10722-008-9373-3.

Yan, J., Su, P., Wei, Z., Nevo, E., and Kong, L. 2017. Genome-wide identification, classification, evolutionary analysis and gene expression patterns of the protein kinase 
426 gene family in wheat and Aegilops tauschii. Plant Mol. Biol. 95(3): 227-242. doi: 427 10.1007/s11103-017-0637-1.

428 Yang, W.-Y. 1992. A preliminary study on the inieritance of the brittle rachis in 429 hexaploid wheat (T. aestivum L.). J. Sichuan Agric. Univ. 1: 145-148.

430 Yang, W.-Y., Rao, S.-D., and Liang, B.-B. 1993. Research and utilizaiton of Chinese 431 special comom wheat germplase. J. Mianyang Agric. Coll. 10: 15-18.

432 Yang, W.-Y., Yen, C., and Yang, J.-L. 1992a. Cytogenetic study on the origin of some 433 special Chinese landraces of common wheat. Wheat Inform. Serv. 75: 14-20.

434 Yang, W.-Y., Yen, C., and Yang, J.-L. 1992b. The study on origin of special kind of 435 hexaploid wheats from China. J. Sichuan Agric. Univ. 10(1): 12-18.

436

437 

439

\begin{tabular}{|c|c|c|c|}
\hline Species & Genome & Number & Accession \\
\hline T. urartu ${ }^{\dagger}$ & $\mathrm{AA}$ & 2 & PI428270, PI428276 \\
\hline T. beoeticum ${ }^{\dagger}$ & AA & 2 & AS261, AS262 \\
\hline T. monococcum ${ }^{\dagger}$ & AA & 5 & $\begin{array}{l}\text { PI362610, PI377666, PI377671, } \\
\text { PI393496, PI427959 }\end{array}$ \\
\hline T. timopheevii ${ }^{\dagger}$ & AAGG & 1 & $\mathrm{AS} 275$ \\
\hline T. araraticum $^{\dagger}$ & AAGG & 3 & PI427300, PI427302, PI427408 \\
\hline T. polonicum & AABB & 4 & $\begin{array}{l}\text { PI254214, PI254215, PI266846, } \\
\text { PI272564 }\end{array}$ \\
\hline T. persicum & AABB & 4 & $\begin{array}{l}\text { PI94752, PI182471, PI251914, } \\
\text { PI272521 }\end{array}$ \\
\hline T. turanicum ${ }^{\ddagger}$ & AABB & 2 & PI124494, PI352514 \\
\hline T. paleo-colchicum ${ }^{\dagger}$ & $\mathrm{AABB}$ & 1 & PI349050 \\
\hline T. durum ${ }^{\dagger}$ & $\mathrm{AABB}$ & 1 & AS2247 \\
\hline T. dicoccoides ${ }^{\dagger}$ & AABB & 3 & AS838, AS847, PI470947 \\
\hline T. dicoccum ${ }^{\dagger}$ & AABB & 5 & $\begin{array}{l}\text { PI94614, PI94666, PI94670, } \\
\text { PI94675, PI113961 }\end{array}$ \\
\hline T. zhukovskyi ${ }^{\S}$ & $\mathrm{AAA}^{\mathrm{m}} \mathrm{A}^{\mathrm{m}} \mathrm{GG}$ & 2 & PI296968, PI355706 \\
\hline T. $m a c h a^{\S}$ & AABBDD & 2 & PI355513, PI355514 \\
\hline T. compactum ${ }^{\dagger}$ & AABBDD & 4 & $\begin{array}{l}\text { AS352, PI129528, PI157920, } \\
\text { PI211701 }\end{array}$ \\
\hline T. spelta ${ }^{\dagger}$ & AABBDD & 3 & AS328, CItr13967, PI15865 \\
\hline T. vavilovii & AABBDD & 3 & AS345, AS346, PI326319 \\
\hline T. sphaerococum & AABBDD & 2 & AS348, AS349 \\
\hline T. petropavlovishyi $i^{\ddagger}$ & AABBDD & 1 & AS359 \\
\hline T. yunnanense $e^{\ddagger}$ & AABBDD & 1 & AS338 \\
\hline T. tibetanum & AABBDD & 1 & Q1028 \\
\hline
\end{tabular}


Table 2 Classification of the amplified sequences in this study

444

\begin{tabular}{|c|c|c|c|c|c|}
\hline Type & $\begin{array}{l}\text { Num. } \\
\text { of sp. }\end{array}$ & $\begin{array}{l}\text { Num. } \\
\text { of ac. }\end{array}$ & $\begin{array}{l}\text { Length } \\
\text { (bp) }\end{array}$ & Species & Accession \\
\hline I & 1 & 1 & 2009 & T. petropavlovishyi & AS359 \\
\hline \multirow[t]{7}{*}{ II } & 7 & 16 & 2069 & T. persicum & PI94752, PI251914, PI272521 \\
\hline & & & & T. zhukovskyi & PI296968 \\
\hline & & & & T. compactum & PI129528, PI211701 \\
\hline & & & & T. macha & PI355513, PI355514 \\
\hline & & & & T. spelta & AS328, PI15865, CITR13967 \\
\hline & & & & T. sphaerococum & AS348, AS349 \\
\hline & & & & T. vavilovii & AS345, AS346, PI326319 \\
\hline \multirow[t]{5}{*}{ III } & 5 & 6 & 2070 & T. dicoccoides & AS838 \\
\hline & & & & T. persicum & PI182471 \\
\hline & & & & T. compactum & PI157920, AS352 \\
\hline & & & & T. yunnanense & AS338 \\
\hline & & & & T. tibetanum & Q1028 \\
\hline \multirow[t]{2}{*}{ IV } & 2 & 7 & 2071 & T. dicoccoides & AS847, PI470947 \\
\hline & & & & T. dicoccum & PI94614, PI94666, PI94670, PI94675, PI113961 \\
\hline $\mathrm{V}$ & 1 & 2 & 2073 & T. polonicum & PI254214, PI254215 \\
\hline \multirow[t]{2}{*}{ VI } & 2 & 4 & 2076 & T. durum & AS2247 \\
\hline & & & & T. araraticum & PI427300, PI427302, PI427408 \\
\hline VII & 1 & 2 & 2077 & T. urartu & PI428270, PI428276 \\
\hline \multirow[t]{2}{*}{ VIII } & 2 & 6 & 2087 & T. beoeticum & AS261, AS262 \\
\hline & & & & T. monococcum & PI427959, PI362610, PI377666, PI377671 \\
\hline IX & 1 & 1 & 2088 & T. monococcum & PI393496 \\
\hline $\mathrm{X}$ & 1 & 1 & 2180 & T. zhukovskyi & PI355706 \\
\hline \multirow[t]{2}{*}{ XI } & 2 & 5 & 2449 & T. polonicum & PI272564, PI266846, PI124494, PI352514 \\
\hline & & & & T. paleo-colchicum & PI349050 \\
\hline XII & 1 & 1 & 2552 & T. timopheevii & $\mathrm{AS} 275$ \\
\hline
\end{tabular}


447

448

449

450

451

452

453

454

455

456

457

458

459

460

461

462

463

464

465

466

467

468

469

470

471

472

473

474
Figure legends

Fig. 1 Isolation of breakpoint regions of the $4 \mathrm{AL}$ junction for the $4 \mathrm{AL} / 5 \mathrm{AL}$ translocation in Triticum. A Locations of WD3L, PEMIL, P1 (F1 and R1), P2 (F2 and R2) and breakpoint. B PCR bands (1500-1800 bp) amplified by primer P1. M, DNA Marker; 1 T. dicoccoides AS838; 2 T. dicoccoides AS847; 3 T. dicoccum PI94666; $4 T$. dicoccum PI94670; 5 T. dicoccum PI94675; 6 T. polonicum PI254214; 7 T. polonicum PI254215; 8 T. araraticum PI427302; 9 T. urartu PI428276; 10 T. monococcum PI362610; 11 T. monococcum PI393496; 12 T. polonicum PI266846. C PCR bands (600-900 bp) amplified by primer P1. M, DNA Marker; 1 T. petropavlovishyi AS359; 2 T. dicoccoides AS838; 3 T. compactum PI157920; 4 T. dicoccoides AS847; $5 T$. dicoccoides AS847; 6 T. dicoccum PI94666; 7 T. dicoccum PI94670; 8 T. araraticum PI427302; 9 T. urartu PI428270; 10 T. urartu PI428276; 11 T. beoeticum AS261; 12 T. beoeticum AS262; 13 T. monococcum PI393496; 14 T. zhukovskyi PI355706.

Fig. 2 Bootstrap consensus tree was obtained by 1000 repeat calculation based on 52 breakpoint sequences of the 4AL junction for the 4AL/5AL translocation in Triticum. The branch with bootstrap value of 0 indicates that the sequences vary greatly between the species or accessions.

Fig. 3 Multiple sequence alignments of the breakpoints from the studied 21 species. The blue bar indicates the sequence was similar to sequences on $4 \mathrm{AL}, 4 \mathrm{BL}$ and $4 \mathrm{DL}$. The green bar indicates the sequence was similar to sequences on 4AL, 5BL and 5DL. Gray bar indicates the sequence cannot find hits in BLAST analysis. And red bars indicates the sequence had similarity to sequences on $4 \mathrm{AL}$. 
(A)

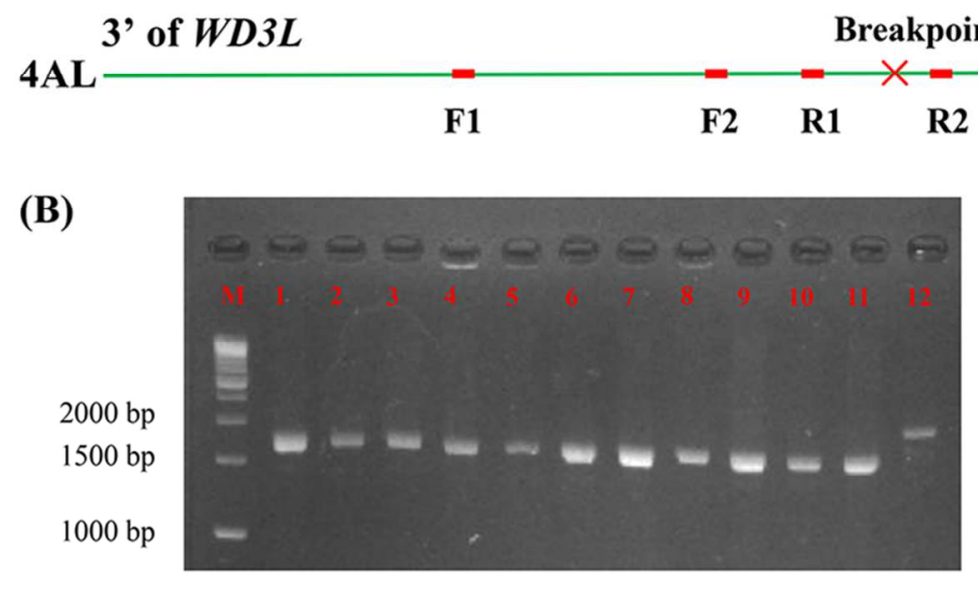

(C)

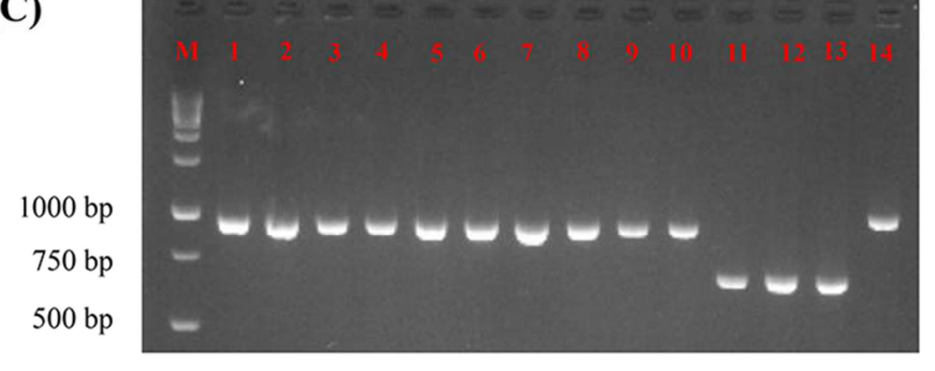

$47 \times 38 \mathrm{~mm}(600 \times 600 \mathrm{DPI})$ 


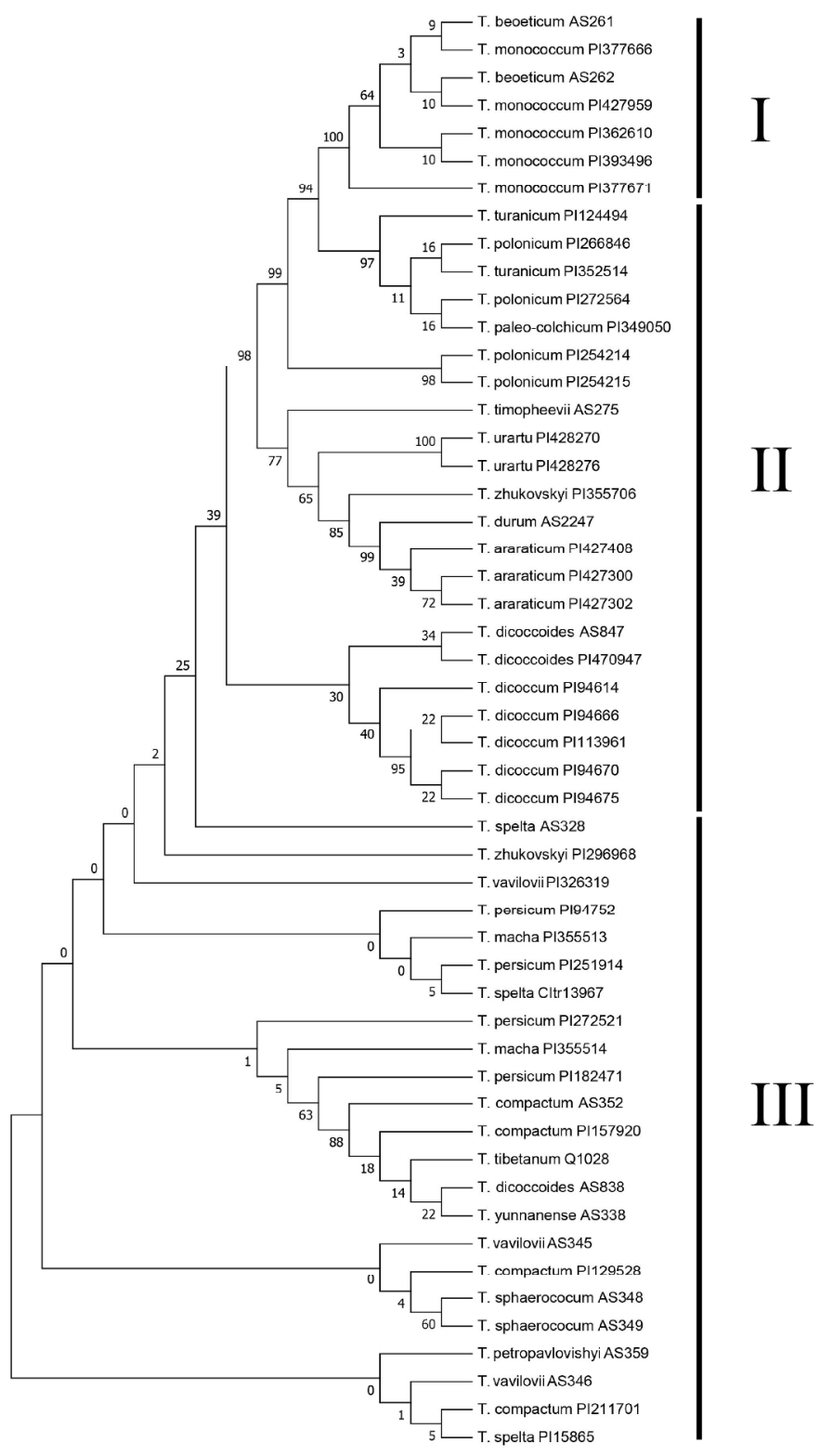

$159 \times 281 \mathrm{~mm}(300 \times 300 \mathrm{DPI})$ 


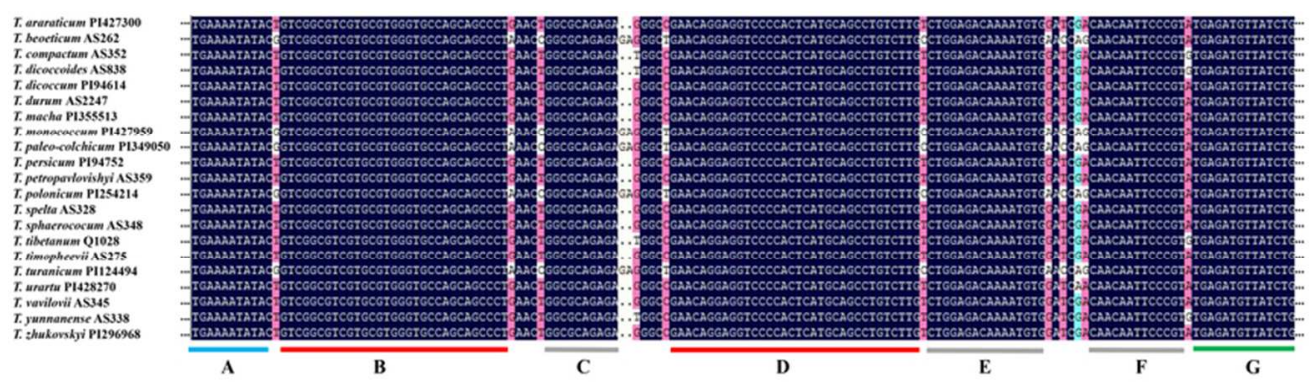

$72 \times 20 \mathrm{~mm}(300 \times 300 \mathrm{DPI})$ 